\title{
"Perder la propia identidad". La adolescencia LGTBQA+ frente a la pandemia por COVID-19 y las medidas del estado de alarma en España
}

\author{
R. Lucas Platero Méndez'; Miguel Ángel López Sáez²
}

En marzo y mayo de 2020, en plena pandemia de COVID-19 en España, los medios se han hecho eco de las recomendaciones de expertos que afirmaban que "los niños suponen un riesgo muy elevado de propagación de la epidemia" (Hermoso, 2020), tildándolos de "vectores de contagio". Aunque cada vez hay más evidencias que cuestionan esta afirmación (Molloy y Bearer, 2020), el Gobierno español ha confinado a ocho millones de niñas, niños y adolescentes (INE, 2017) en sus hogares (Orden SND/370/2020). Estas medidas de confinamiento han sido muy duras ${ }^{3}$, en comparación con las de otros países del entorno, evidenciando la gran brecha social en función de los recursos familiares (Martínez, Rodríguez y Velásquez, 2020).

En un clima tildado de "anti-niños" (Flecha, 2020), este etiquetamiento ha hecho que no se preste atención al riesgo de tales medidas de confinamiento para infancia y juventud incluidas en el estado de alarma; un confinamiento que en estudios previos están ligados a la aparición del estrés postraumático (Sprang y Silman, 2013). No ha sido hasta las medidas de desescalada del confinamiento, en mayo de 2020, cuando se han ofrecido medidas dirigidas a la infancia y juventud, con horarios y espacios restringidos

Son medidas que tienen un impacto particularmente negativo para la infancia y juventud que tienen sexualidades e identidades de género no normativas, que de entrada sabemos ya viven unas condiciones de vida que están atravesadas por un mayor estrés y ansiedad que el resto debido a la falta de apoyos (Platero y Ceto, 2007; Coll, Bustamante y Missé, 2009; Puche, Moreno y Pichardo, 2013; Platero, 2014; Missé, 2018), tienen un mayor riesgo de suicidio y de consumo de sustancias (Frost, Meyer y Schwartz, 2016). Una vulnerabilidad que es más pronunciada en quienes son jóvenes trans o personas no binarias (Platero 2014; Green, Dorison y Price-Feeney, 2020).

\section{Metodología.}

- Participantes. En el reciente estudio que elaboramos sobre los impactos psicosociales de la pandemia del COVID-19 en personas LGTBQA+ en España, con datos recogidos entre el 9 de marzo y el 9 de mayo de 2020, se obtuvieron 2832 respuestas. Un total de 93 participantes entre 13 y 17 años de edad respondieron a la encuesta $(M=16.17, D E=.90, M d n=16)$. Aproximadamente el $35.5 \%$ de la muestra se identificó como mujeres cis, el 9.7\% como hombres cis, el $34.4 \%$ como hombres trans, el 8,6\% como mujeres trans y el $11.8 \%$ como género no binario. El $60.2 \%$ de los participantes se declaró bisexual, el $21.5 \%$ homosexual, el $9.7 \%$ como heterosexual y el $8.7 \%$ dentro de otras orientaciones. (como pansexual, antrosexual, asexual, etc.).

- Procedimiento. Los participantes fueron reclutados a través de redes sociales, correos electrónicos a asociaciones LGTBI y servicios públicos de atención a personas LGTBI y medios de comunicación (eldiario.es, elsalto.com y Pikara Magazine entre otros). Con este método de reclutamiento se utilizó una técnica de bola de nieve (Charmaz, 2014). A los participantes se les proporcionó una dirección web para acceder a la encuesta electrónica. Y antes de hacer la encuesta, debían leer un consentimiento informado.

\footnotetext{
Dpto. Psicología Social, Universitat Autónoma de Barcelona. Miembro de los equipo de investigación AFIN y Fractalidades de la Investigación Crítica.

E-mail: Lucas.Platero@uab.cat

Profesor visitante, Universidad Rey Juan Carlos

E-mail: miguel.lopez.saez@urjc.es

Desde el día 14 de marzo se suspendieron las clases, y no han podido salir de casa (excepcionalmente acompañando a una persona adulta en salidas autorizadas). Los menores de 14 años no han podido salir a pasear hasta el 26 de abril, una vez al día, a una distancia de un kilómetro del domicilio y acompañados de un adulto, de 9 a 21h. Para evitar aglomeraciones y en poblaciones de más de 5000 habitantes, el 2 de mayo se establecieron horarios por edades, donde la juventud de más de 14 años por fin pudo salir en la franja adulta (6-10h y 20-23h); mientras los menores de 14, para dar paseos, comenzaron otro horario, de $12-19 \mathrm{~h}$. 


\section{Resultados}

El análisis preliminar sobre estos datos arroja informaciones relevantes sobre el efecto específico de la pandemia en la adolescencia con sexualidades e identidades de género no normativas. Se están enfrentado a grandes riesgos para su salud psicosocial, debido a la convergencia de una serie de factores; un riesgo que además es variable en función de las desigualdades interseccionales que atraviesan sus vidas (clase social, discapacidad, género, procedencia nacional y étnica, y otras) y que determinan su calidad de vida.

Dichos factores son:

1. Se han reducido las interacciones sociales positivas con respecto a su identidad o expresión sexual y de género, debidas a las medidas del confinamiento, aislamiento y distancia social, que restringen su vida al contexto familiar del hogar. En sus hogares pueden estar viviendo una mayor monitorización de sus comportamientos y expresiones de género, pudiendo surgir situaciones con mayor violencia, que de otra manera podrían aparecer más atenuadas, desplazadas a otros contextos o pasar desapercibidas. Tengamos en cuenta que a pesar de ser mucho más visibles que en el pasado y recibir el apoyo de asociaciones, no en todos los hogares se acepta a esta infancia y juventud que desafía las normas de género, que tiene expresiones de género no normativas y que se declara LGTBQA+. De hecho, un 62,4\% sienten tener que ocultar su orientación y/o identidad en su hogar actual, en algún grado.

Se producen experiencias familiares de apertura sobre sus vivencias, que puede ser experimentada por las personas adultas con sorpresa, no sabiendo cómo abordarlas, en ocasiones con una escucha condicionada y la incertidumbre frente a una identidad sexual calificada de temprana, y por tanto, no resuelta (Castañeda, 2014). En este sentido, la implicación familiar resulta insatisfactoria para el 81,7\% de jóvenes, que expresa en algún grado una ausencia de apoyos para la escucha, la comprensión y el afecto.

2. El aumento de interacciones sociales negativas con respecto a su identidad, debido a las mencionadas limitaciones del contexto familiar no son las únicas. La aparición de una sensación subjetiva de soledad está vinculada a la necesidad adolescente de pertenencia social (Green, Dorison y Price-Feeny, 2020), así como de singularidad de sus vivencias ("esto solo me pasa a mi") que aluden a su corporalidad, sus identidades no normativas y de necesidad de vínculo con iguales LGTBQA+. Expresan no tener a quién recurrir en caso de necesitar ayuda, en el $64,5 \%$ de las ocasiones. Incluso cuando incluyen a sus amistades, recurren a ellas totalmente solo en un $41,9 \%$ de las veces.

Las interacciones dan sentido a la identidad propia, de ahí el título de este artículo, tomado de una afirmación que hizo un joven trans gallego sobre sus vivencias en este período (Moledo Ucha, 2020). Desde el punto de vista del desarrollo evolutivo, la conexión con otras personas durante la adolescencia es fundamental, un factor de salud clave para esta infancia y juventud que tiene que lidiar con no contar necesariamente con el apoyo social que necesitan (Platero, 2014). Son interacciones que pueden aliviar el estrés, reducir la depresión y mejorar su bienestar (Cohen, 2004), y en particular, vincularse a otras personas LGTBQA+ sirve para poder enfrentarse al estigma de la depresión o el suicidio (Kaniuka et al., 2019).

El vínculo con iguales les ayuda a dar sentido a lo que están viviendo, escapar al juicio de valores adultocentrista. El 69,9\% de la población adolescente participante afirma que resulta esencial el apoyo entre iguales LGTBQA+. Además, afirma recibir apoyo total por parte de iguales LGTBQ (el 57\% de la juventud LGTBQA+), mientras que el mismo tipo de apoyo total por parte de aliados cisheterosexuales resulta menor (un 39,8\%).

La búsqueda de estas interacciones se puede desplazar a los contextos online donde pueden cuestionar la heteronomatividad (De-Ridder y Van-Bauwel, 2013). En la red pueden mostrar públicamente sus identidades ante un pequeño público, antes de hacerlo con sus familias, y además pueden iniciarse incluso en cierto activismo online (Jenzen, 2017). Estos espacios online, sin supervisión adulta, ofrecen cierta sensación de libertad, pero también facilitan exponerse a otros riesgos potenciales, como son las agresiones LGTBfóbicas online. Si en 2018 el Observatorio Madrileño contra la Homofobia, Transfobia y Bifobia indicaba 41 agresiones a través de internet, en los últimos días los casos de menores que las sufren parecen acumularse y el hashtag \#damelapalizaamítambien se está usando para visibilizarlas (Shangay, 2020). En ese sentido, el 73,1\% de la población adolescente participante ha observado en algún grado un incremento de las agresiones a través de internet.

3. El aumento de malestares, y la búsqueda de mecanismos para hacerles frente, son consecuencias derivadas de la difícil situación que viven en su cotidianeidad confinada. La ausencia de redes de apoyo supone un riesgo para el incremento de factores estresantes (Frost, Meyer y Schwartz, 2016). Lo que se traduce en mayores tasas de ansiedad y depresión (Bissonette y Szymanski, 2019) y de consumo de drogas (Gayles y Garofalo, 2019), entre otros. A nivel nacional, el riesgo de suicidio se multiplica por tres (Generelo, Garchitorena, Montero e Hidalgo, 2012). Por lo que puede resultar de especial importancia prestar atención a sentimientos asociados a sentirse una carga o un peso para los demás en algunos adolescentes, como señala el $67,7 \%$ de la juventud LGTBQA+ participante al sentirse que contribuyen a empeorar las cosas para su gente cercana, en algún grado. Y para hacer frente a esas situaciones, un $14 \%$ de las personas adolescentes participantes plantea el consumo de alcohol, tabaco e incluso antidepresivos como fórmulas para evadirse, evitar sentimientos negativos o dejar de estar avergonzados. 
4. La falta de atención específica desde sus centros escolares a la sexualidad, así como desde los recursos existentes en los espacios prioritarios de socialización (Pichardo y De Stéfano, 2015; Coll, Bustamante y Missé, 2009; Platero y Ceto, 2007). Esta ausencia ya existía previamente, tratando la sexualidad como un tema secundario, tabú o controvertido. Bajo la argumentación de la protección a este grupo etario por su estatus como menores de edad, están aumentando discursos morales conservadores sobre los derechos sexuales de la infancia, y que han llevado a monitorizar y facilitar la prohibición de la educación afectivo-sexual, con la aparición del mal llamado "Pin Parental" en algunas comunidades autónomas. Estas barreras sobre la educación sexual tienen un claro impacto negativo en su salud (Coll, Bustamante y Missé, 2009; Platero, 2014).

Por la urgencia de la situación, incluso los centros escolares más proactivos con la educación sexual han tenido que centrarse más en navegar en la transición a la enseñanza online (Martín-Arroyo, 2020), que en ser el sostén de la juventud frente la discriminación por LGTBQAfobia, o en sus problemas de salud sexual y reproductiva.

5. Un estrés añadido importante, debido a la incertidumbre económica y social viven sus familias, que se enfrentan a numerosas pérdidas de empleos y la aprobación de ERTES en sus empresas, que no tienen apoyos para conciliar su vida laboral teletrabajando y de cuidados. Además, existe una incertidumbre específica sobre la infancia y adolescencia sobre su futuro inmediato y a medio plazo (Martínez; Rodríguez y Velásquez, 2020), con el anuncio del Gobierno la continuidad de las medidas de distancia social, restricciones de espacios y horarios específicos, así como el cierre de los centros escolares, la posibilidad de vuelta a la escuela en septiembre con grupos reducidos y en días alternos (Zafra, 2020).

6. El parón en la actividad la administración durante el Estado de Alarma puede ser un varapalo importante para jóvenes que llevan mucho tiempo esperando para poder cambiar su documentación, para que sea acorde a su sexo, o poder acceder a tratamientos hormonales (Moledo-Ucha, 2020). Este es un tiempo en pausa, que detiene los procesos de reconocimiento que son vitales para sostener la esperanza en que su vida será más vivible.

\section{Conclusiones}

Los datos recogidos aún tienen que ser analizados en profundidad y han de completarse con otro tipo de datos de corte más cualitativo, pero podemos anticipar algunas ideas relevantes. Hay factores psicosociales específicos que atraviesan las vidas de la juventud con sexualidades, identidades y expresiones de género no normativas durante la pandemia del COVID-19, que podrían ser más intensos para quienes viven en España por el tipo de medidas impuestas (confinamiento, restricción de movilidad, suspensión de clases, etc.).

De hecho, hemos identificado seis factores psicosociales específicos para la adolescencia LGTBQA+: 1) reducción de interacciones sociales positivas con respecto a su identidad; 2) aumento de interacciones sociales negativas con respecto a su identidad; 3) aumento de malestares ligados a la incertidumbre; 4) búsqueda de mecanismos para hacerles frente; 5) falta de atención específica a la sexualidad en sus centros escolares; y 6) parón en la actividad la administración durante el estado de elarma. Dichos factores pueden orientar sobre cómo hacer políticas públicas sobre la infancia y juventud, cómo poder mejorar la atención específica, y ayudar a pensar qué consecuencias pueden tener algunas medidas futuras en posibles situaciones de confinamiento.

Con estos datos, se subraya la importancia de tener en cuenta no solo a la infancia y juventud cuando se hacen medidas sobre la salud pública, sino a quienes tienen sexualidades e identidades género no normativas, anticipando medidas concretas y específicas para la prevención de daños en la salud psicosocial.

\section{Agradecimientos}

Esta investigación y este artículo no hubieran sido posibles sin el apoyo de los medios de comunicación, las instituciones, y movimientos sociales que apoyan los derechos sexuales en el Estado español.

\section{Financiación}

El premio Emma Goldman, Flax Foundation, y la financiación del Contrato Juan de la Cierva han hecho posible este artículo.

\section{Referencias bibliográficas}

Bissonette, D., Szymanski, D. M. (2019). Minority stress and LGBQ college students' depression: Roles of peer group and involvement. Psychology of sexual orientation and gender diversity, 6(3), 308. doi:10.1037/sgd0000332

Castañeda, C. (2014). Child. Transgender Studies Quaterly, 1(1), 59-61.

Cohen, S. (2004). Social Relationships and Health. American Psychologist, 59(8), 676-684. 
Coll, G., Bustamante, G., Missé M. (2009). Transitant per les fronteres del gènere: Estratègies, trajectòries i aportacions de joves trans, lesbianes i gais. Barcelona: Secretaria de Joventut, Generalitat de Catalunya.

De-Ridder, S., Van-Bauwel, S. (2013). Commenting on pictures: Teens negotiating gender and sexualities on social networking sites. Sexualities, 16(5-6), 565-586.

Frost, D. M., Meyer, I. H., Schwartz, S. (2016). Social support networks among diverse sexual minority populations. American Journal of Orthopsychiatry, 86(1), 91.doi:10.1037/ort0000117

Gayles, T. A., Garofalo, R. (2019). Exploring the Health Issues of LGBT Adolescents. En J. Schneider, V. Silenzio, L. Erickson-Schroth (Eds.), The GLMA Handbook on LGBT Health (pp. 133-154). California: Praeger.

Generelo, J., Garchitorena, M., Montero, P., Hidalgo, P. (2012). Acoso escolar homofóbico y riesgo de suicidio en adolescentes y jóvenes $L G B$. Madrid: COGAM/FELGTB (en línea).

http://www.felgtb.org/rs/1584/d112d6ad-54ec-438b-9358-4483f9e98868/91c/filename

Goosby, B. J., Bellatorre, A., Walsemann, K. M., Cheadle, J. E. (2013). Adolescent loneliness and health in early adulthood. Sociological Inquiry, 83(4), 505-536. https://doi.org/10.1111/soin.12018

Green, A., Dorison, Samuel, Price-Feeny, M. (2020). Implications of COVID-19 for LGBTQ Youth Mental Health and Suicide Prevention. Trevor Project.

Flecha, A. (2020). No son vectores, son niños y niñas. ElSalto.com, 23/04/2020.

Hermoso, B. (2020). Los niños suponen un riesgo muy elevado de propagación de la epidemia. El País, 20/03/2020.

Instituto Nacional de Estadística (2017). La juventud en cifras (en línea). http://www.injuve.es/observatorio/demografia-e-informacion-general/juventud-en-cifras-poblacion-marzo-2017

Jenzen, O. (2017). Trans youth and social media: moving between counterpublics and the wider web. Gender, Place \& Culture, 24(11), 1626-1641.

Kaniuka, A., Pugh, K. C., Jordan, M., Brooks, B., Dodd, J., Mann, A. K., Williams, S., Hirsch, J. K. (2019). Stigma and suicide risk among the LGBTQ population: Are anxiety and depression to blame and can connectedness to the LGBTQ community help? Journal of Gay \& Lesbian Mental Health, 23(2), 205-220. doi:10.1080/19359705.2018.1560385

Martín-Arroyo, J. (2020, 2 de abril). Tres semanas a destajo para dar clase de otra manera. Elpais.com. https://elpais.com/sociedad/2020-04-02/tres-semanas-a-destajo-para-dar-clase-de-otra-manera.html

Martínez Muñoz, M.; Rodríguez Pascual, I., Velásquez Crespo, G. (2020). Infancia Confinada. ¿Cómo viven la situación de con namiento niñas, niños y adolescentes? Madrid. Infancia Confinada y Enclave de Evaluación.

Missé, M. (2018). A la conquista del cuerpo equivocado. Barcelona y Madrid: Egalés.

Moledo Ucha, A. (2020). Estanse a dar conflitos importantes. Diario de Pontevedra, 28/04/2020.

Molloy, E.J., y Bearer, C.F. (2020). COVID-19 in children and altered inflammatory responses. Pediatric Research . doi:10.1038/ s41390-020-0881-y

Observatorio madrileño contra la LGTBIfobia (2018). Informe sobre delitos de odio por LGTBfobia en la Comunidad de Madrid 2018. Madrid (en línea). http://www.contraelodio.org/informes/2018/informe_obsmad_comunidad_madrid_2018.pdf

Orden SND/370/2020, de 25 de abril, sobre las condiciones en las que deben desarrollarse los desplazamientos por parte de la población infantil durante la situación de crisis sanitaria ocasionada por el COVID-19. BOE, 116, de 25 de abril de 2020 , 30361-30364.

Platero, R. L. (2014). Trans*exualidades. Acompañamientos, factores de salud y recursos educativos. Barcelona: Bellaterra.

Platero, R., Ceto, E. (2007). Herramientas para combatir el bullying homofóbico. Madrid: Talasa.

Pichardo, J. I., De Stéfano, M., (Eds.). (2015). Diversidad sexual y convivencia: una oportunidad educativa. Madrid: Universidad complutense de Madrid (en línea). https://eprints.ucm.es/34926/1/Diversidad y convivencia 2015 FINAL.pdf

Puche, L., Moreno, E., Pichardo, J. I. (2013). Adolescentes transexuales en las aulas. Aproximación cualitativa y propuestas de intervención desde la perspectiva antropológica. En O. Moreno, L. Puche, (Eds.), Transexualidad, adolescencias y educación. Miradas multidisciplinares (pp. 189-265). Barcelona y Madrid: Egalés.

Shangay (2020, 9 de mayo). Ana Milán denuncia una nueva agresión homófoba en Instagram. Shangay.com (en línea). https:// shangay.com/2020/04/20/ana-milan-denuncia-una-nueva-agresion-homofoba-en-instagram/

Sprang, G., Silman, M. (2013). Posttraumatic Stress Disorder in Parents and Youyh After Health-Related Disasters. Disaster Medicine and Public Health Preparedness, 7(1), 105-110.

Zafra, I. (2020, 6 de mayo). 15 alumnos por aula, sin espacio ni profesorado suficientes. Elpais.com. https://elpais.com/sociedad/2020-05-05/15-alumnos-por-aula-sin-espacio-ni-profesorado-suficientes.html 